\title{
Financial Contagion Simulation through Modelling Behavioural Characteristics of Market Participants and Capturing Cross-Market Linkages
}

\author{
Antoaneta Serguieva ${ }^{1}$, Fang Liu², Paresh Date \\ ${ }^{1}$ Department of Computer Science, University College London, London WC1E 6BT \\ ${ }^{2}$ Brunel Business School, Brunel University, Uxbridge UB8 3PH \\ ${ }^{3}$ Department of Mathematical Sciences, Brunel University, Uxbridge UB8 3PH
}

\begin{abstract}
Over the past two decades, financial market crises with similar features have occurred in different regions of the world. Unstable cross-market linkages during financial crises are referred to as financial contagion. We simulate the transmission of financial crises in the context of a model of market participants adopting various strategies; this allows testing for financial contagion under alternative scenarios. Using a comprehensive approach, we develop an agentbased multinational model and identify factors contributing to contagion. Although contagion has been investigated in the financial literature, it has not yet been studied extensively through computational intelligence techniques. The first steps in that direction are taken in (Serguieva, Wu 2008; Caporale, Serguieva, Wu 2008; Caporale, Serguieva, Wu 2009; Liu, Serguieva, Date 2010). We extend these studies and introduce GARCH model and Clayton copula to capture better markets interdependence and to improve the evolutionary optimization technique. Our model further comprises four rather than three types of traders: technical, game, herd, and noise, traders respectively. The different types of traders use different computational strategies to make now three rather than two kinds of decisions : "buy", "sell", or "hold" decisions. Our simulations shed light on parameter values and characteristics which can be exploited in further research to detect contagion at an earlier stage, hence recognizing financial crises with the potential to destabilize cross-market linkages.
\end{abstract}

\section{Introduction}

A series of historical financial crises, such as Mexico in 1987, Asia in 1997, and Russia in 1998, all share a common feature - problems spread from one country to others due to the cross-market linkages. If the cross-market linkages stay stable then the crises is transferred through interdependence, and the recovery follows the recovery of the underlying economic reason in the country of origin. When the cross-market linkages get destabilized due to the crisis, then the crisis starts "feeding on itself" and the recovery of the underlying economic reason is not sufficient to get control of the crisis. A more comprehensive strategy with international involvement is required. The second type of crisis exhibits the phenomenon called 'financial contagion'.

There is no conclusive technical definition of the financial contagion phenomenon described above. Most research papers identify contagion by analyzing the change in the variance-covariance matrix during the period of market turmoil. King and Wadhwani (1990) first test the correlations between the US, UK and Japan, during the US stock market crash in 1987. Boyer (1997) finds significant increases in correlation during financial crises, and reinforces a technical definition of financial contagion as a correlation breakdown during the crash period. Forbes and Rigobon (2002) define contagion as "a significant increase 
in cross-market linkages after a shock to a group of countries". In their work, the term "interdependence" is used as the alternative to "contagion". Interdependence leads to common price movements during periods both of stability and turmoil.

In the past two decades, many studies (e.g. Kaminsky, Lizondo, Reinhart 1998; Kaminsky 1999) developed early warning systems focused on the origins of financial crisis rather than on financial contagion. Other works (e.g. Forbes, Rigobon 2002; Caporale, Cipollini, Spagnolo 2005) focused on studying contagion compared to interdependence. Rodriguez (2006) finds the change of tail dependence is a warning sign for financial contagion. Yang and Bessler (2006) use vector auto-regression analysis to explore the financial contagion pattern characters. Cipollini and Kapetanios (2009) use principal components analysis to find out indicators of contagion. In this paper, we model and simulate the transmission of financial crises through the behavior of market players and their various strategies, using an integrated approach that involves a mixed-game, a multinational agent-based mode, genetic programming (GP), and Clayton Copula. Our multinational model is developed to suit analyzing financial contagion; it is composed of four types of traders - technical, fundamental, herd and noise traders. A technical trader is a trader who makes decisions based on the technical analysis of price charts. Technical traders analyze price charts to develop theories about the direction in which the market is likely to move. In an artificial market, technical traders are usually modelled through GP. Fundamental traders make buy or sell decisions based on companies' fundamentals such as P/E ratio, etc. In our model, we use game players as a proxy to fundamental traders, due to the limitation of available data. Game theory is a branch of applied mathematics and economics. The so-called "minority game", as a further development of game theory, is especially useful to simulate real financial markets (Lebaron 2006). Herd traders, as important as technical and fundamental traders when describing market behavior, are the ones who make decisions following the prevailing behaviour, regardless of other factors and market fundamentals. Herd behaviour has been identified as a major factor behind contagion (Cont and Bouchaud 2000). Finally, noise traders are stock traders whose decisions are irrational and erratic: their presence in financial markets can cause prices and risk levels to diverge from expected levels even if all other traders are rational (De Long et al 1990)

Building a multinational minority-game or mixed-game model of financial contagion has been considered in (Serguieva, Wu 2008; Caporale, Serguieva, Wu 2008; Caporale, Serguieva, Wu 2009). In (Liu, Serguieva, Date 2010), the authors build on these previous studies and extend the number of types of traders and kinds of trading decisions In this paper particularly, we develop a comprehensive model comprising four types of traders: technical, herd, game, and noise traders, respectively. Furthermore, we consider three kinds of decisions: buy, hold and sell, and we also use a different price formation and assets allocations approach. Finally, we introduce the GARCH model and Clyton-copula approach to better capture cross market linkages 


\section{Research Method}

Real financial markets are composed of different types of participants who interact through asset trading. A market player $i$ generally holds two types of assets:

a risky asset, denoted by $h_{i}(t)$

cash, denoted by $c_{i}(t)$

The number of technical, game, herd and noise traders is denoted as $N_{T}, N_{F}, N_{H}, N_{N}$, respectively. The notation $P(t)$ stands for the share price at time $t$. The initial conditions include 10 shares and $£ 10,000$ cash available to each player. At any step in time, a trader buys or sells certain number of assets according to their own trading rules.

\subsection{Price Formation and Assets Allocation}

The price formation mechanism that we use in this paper is similar to that in (Martinez-Jaramillo, Tsang 2009) who based their work on price determination proposed in (Cont, Bouchaud 2000; Jefferies, Hart, Hui, Johnson 2001, Farmer 1998). Thus, the price is calculated here with the following formula:

$\operatorname{Price}(t)=\operatorname{Price}(t-1)+D(t) / \sigma$

where $\sigma$ is an important parameter representing the market sensitivity to the order imbalance. $\mathrm{D}(t)$ is the sum of all the individual decisions.

\subsection{Single Market Model}

\subsubsection{Traders}

As noted above, in our model, we classify the market players into four categories:

A) Technical traders: Technical analysis is a key feature of our model. This group of traders presents the richest range of behaviors. Technical traders use GP to develop trading rules, and each individual technical trader is represented by a different decision tree. The basic elements of such decision trees are rules and forecast values. A single rule is made up with a combination of three technical indicators, one rational operator such as "greater than" or "less than or equal to", and a real value threshold. The three technical indicators are moving average (MA), trading breakout (TRB), and volatility (VOL). A single rule interacts with other rules in one decision tree through logical operators such as "or", "and", "not" and "if-then-else", as shown in Figure 1 presenting an example of a decision tree. The root node is always an "if-then-else" node (ITE); an ITE node has two children, each of which could be either a decision node or another "ifthen-else" node. The code following Figure 1 shows how the decision rule logic is derived from the decision tree. 


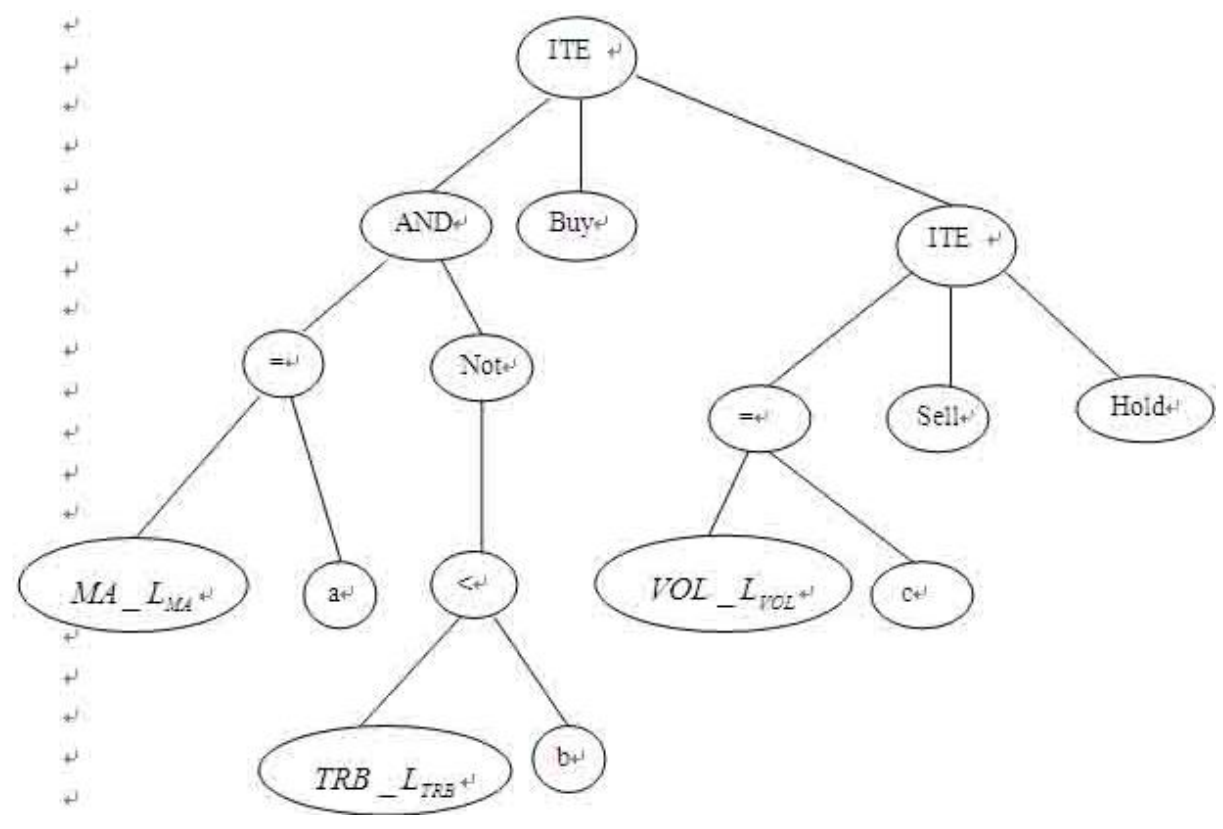

Fig.1. Example of a decision tree

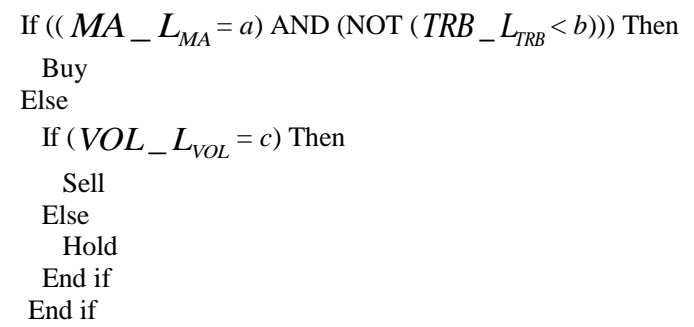

In the above calculations, parameters a, b, c, $L_{V O L}, L_{M A}$ and $L_{T R B}$ are set fixed as in MartinezJaramillo and Tsang (2009) because they are the way in which they are used by practitioners of technical analysis. The Moving Average (MA) indicator is defined as:

$M A\left(L_{M A}, t\right)=\frac{\operatorname{Price}(t)-\left(\frac{1}{L} \sum_{i=1}^{L_{M A}} \operatorname{Price}(t-i)\right)}{\frac{1}{L} \sum_{i=1}^{L_{M A}} \operatorname{Price}(t-i)}$

The Trading Breakout (TRB) indicator is defined as:

$\operatorname{TRB}\left(L_{T R B}, t\right)=\frac{\operatorname{Price}(t)-\max \left(\operatorname{Pr} i c e(t-1), \operatorname{Price}\left(t-L_{T R B}\right)\right)}{\max \left(\operatorname{Pr} i c e(t-1), \operatorname{Pr} i c e\left(t-L_{T R B}\right)\right)}$

The Volatility (VOL) indicator is defined as:

$\operatorname{Vol}\left(L_{V O L}, t\right)=\frac{\sigma\left(\operatorname{Price}(t-1), \cdots \operatorname{Price}\left(t-L_{V O L}\right)\right)}{\frac{1}{L_{V O L}} \sum_{i=1}^{L_{V O L}} \operatorname{Price}(t-i)}$ 
A.1) Co-evolutionary GP setup for technical traders: The basic decision tree for a technical trader is introduced above and we can now build on this to a co-evolutionary nature following a "red queen" principle. The "red queen" principle is originally proposed by Leigh Van Valen (1973) as a metaphor of a co-evolutionary arms race between species. The result is that inevitably, when competing for scarce resources, one party is going to end up the winner controlling the majority of those resources. In our setting, the red queen principle is applied in the form of "red queen retraining", similar to Martinez-Jaramillo and Tsang (2009). When a trader's wealth falls below the average wealth, he/she launches a GP mechanism to evolve the population of rules by retaining half of his/her current population and re-generating the other half randomly. To implement this, after each trade, each trader re-evaluates his trading rules, increasing score for each rule that produced the right decision and reducing score for the rule that gave wrong decisions. During the next trading period, traders make decision following the rule with the highest scores available to them. Note that when apply mutation and crossover, each rule's score stay unchanged. To apply the red queen principle means to reset all scores of a trader's decision trees. At the same time, retain half of his current population and re-generate the other half randomly.

B) Game traders: In our model, these play a mixed game, which is to say that fundamental traders are divided into two groups, one of which plays a minority game and the other plays a majority game. TanakaYamawaki and Tokuka (2006) propose a minority game, where traders take one of two possible actions: buy $(1)$ or sell $(0)$. If the minority side is defined to mean the decision made by a minority of traders, those who end up on the minority side win the game - the price will move in their favour. After each trade is executed, all the traders know, by the way the price has moved, whether the right choice would have been to buy or sell. Also in the model, all agents have their own decision table. This approach is similar to the one applied by Serguieva and Wu (2008). In our model, we add one more choice "hold" (do nothing) to be more realistic. The buy, hold and sell decision are denoted by 1,0 and -1 , respectively.

TABLE 1

Example of a decision table

\begin{tabular}{cccc}
\hline \hline $\begin{array}{c}\text { Historical } \\
\text { string }\end{array}$ & Strategy(1) & Strategy(2) & Strategy(3) \\
\hline$-1,-1$ & +1 & 0 & 0 \\
$-1,0$ & +1 & +1 & 0 \\
$-1,+1$ & 0 & -1 & +1 \\
$0,-1$ & 0 & 0 & 0 \\
0,0 & -1 & 0 & -1 \\
$0,+1$ & +1 & +1 & -1 \\
$+1,-1$ & 0 & +1 & -1 \\
$+1,0$ & -1 & +1 & -1 \\
$+1,+1$ & 0 & -1 & 0 \\
\hline \hline
\end{tabular}


The elements of the Decision table are as follows:

Memory size, $m$

Number of strategies, $k$, included in a decision table

Binary description ( -1 sell, 0 hold, 1 buy)

Huge pool ( $K=3^{3^{m}}$ ) of possible strategies

Table 1 gives an example of a decision table, with $m=2$ and $k=3$. There are $K=19,683$ possible strategies for $m=2$. The decision table of a single agent includes only a few strategies out of these, in our case $k=3$ strategies. The strategy table becomes a baseline for a trader to make decisions. For example, if the historical string is "-1-1", which means the winning decision that happened in the past two trade days, would have been "sell", then strategy one recommends to choose 1 in the current period, which means "buy". But strategy two recommends to hold choosing 0 . To select a strategy and update strategy scores after each trade, traders re-evaluate all strategies thus evolving decision tables; They increase the score for each strategy that produced the winning decision and reduce the score for the strategies that gave wrong decisions. During the next trading period, traders make decision following the strategy with the highest scores available to them. Importantly, all the traders have their own decision tables, and each trader works with different $k$ strategies out of the large strategy pool $K$. The example in Table 1 presents one particular trader. The score $w_{s_{i}}$ for each strategy is calculated as follows:

$$
\begin{aligned}
& w_{s_{i}, t}=w_{s_{i}, t-1}+\alpha_{t} \\
& \alpha_{t}= \begin{cases}1 & \text { right dicision } \\
-1 & \text { wrong decision }\end{cases}
\end{aligned}
$$

where $\alpha_{t}$ is the decision made in time point $t$.

C) Herd traders: These follow the trend of price movements, and the probability of a 'hold' transaction at time $t$ is denoted with $p_{0, t}^{\text {Herd }}$ and calculated as follows:

$$
p_{0, t}^{H e r d}(i)=\frac{1}{1+\left(d\left|\zeta_{t-1}\right|\right)}
$$

where parameter $d$ controls the sensitivity to price change. The probability of a "buy" decision is correspondingly:

$$
p_{1, t}^{\text {Herd }}(i)=\left(1-p_{0, t}^{\text {Herd }}(i)\right) \frac{\exp \left(\zeta_{t-1}\right)}{\exp \left(\zeta_{t-1}\right)+\exp \left(-\zeta_{t-1}\right)}
$$

Here, $\zeta_{t}$ is the overall price change at time $t$. Correspondingly, the probability of a "sell" decision is:

$$
p_{-1, t}^{\text {Herd }}(i)=1-p_{0, t}^{\text {Herd }}(i)-p_{1, t}^{\text {Herd }}(i)
$$


D) Noise traders: These make decisions to buy, sell or do nothing with different probabilities. These probabilities are predefined and remain constant during the whole process of simulation.

\subsection{Multinational Market Model}

We consider a two-nation market for notational and computational simplicity. The results can be generalized to involve more than two countries.

A) Technical traders in a multinational market model: The process by which the technical traders choose the market in which to trade is similar to that followed by game players, (see section B below). All technical players also co-evolve their decision trees.

B) Game players in a multinational market model: In order to simulate the linkage between two markets, the mixed-game-based players are allowed to make investment decisions based on information from both domestic and foreign markets, while investing in the domestic market (Caporale, Serguieva, Wu 2009). We again consider a domestic market $A$ and a foreign market $B$. An agent $i\left(i=1, \cdots N^{A}\right)$ operating in market $A$ has a probability $\left(P_{t}^{D O M, A}(i)\right)$ of choosing an action at time $t$ based on the domestic market:

$$
P_{t}^{\text {DOM }, A}(i)=\frac{\exp \left(\lambda^{A} w_{t}^{A}(i)\right)}{\exp \left(\lambda^{A} w_{t}^{A}(i)\right)+\exp \left(-\lambda^{A} w_{t}^{A}(i)\right)}
$$

where $\lambda^{A}$ is a scale factor. Agents will take actions based on either the domestic or the foreign market. Thus the probability $P_{t}^{F O R, A}(i)$ of an agent in market $A$ choosing an action based on the foreign market is:

$$
P_{t}^{F O R, A}(i)=1-P_{t}^{\text {DOM }, A}(i)=1-\frac{\exp \left(\lambda^{A} w_{t}{ }^{A}(i)\right)}{\exp \left(\lambda^{A} w_{t}^{A}(i)\right)+\exp \left(-\lambda^{A} w_{t}^{A}(i)\right)}
$$

contributing to the linkages between the two markets. Parameter $w_{t}^{A}(i)$ is updated after each period. If a decision based on the foreign market history loses the game, then $w_{t}^{A}(i)=w_{t-1}^{A}(i)+1$. If the action based on the foreign market history wins the game, and the decision based on the domestic market history loses, then $w_{t}^{A}(i)=w_{t-I}^{A}(i)-1$. Therefore, if the decisions based on the domestic and foreign markets are different, $w_{t}^{A}(i)$ is updated (Caporale, Serguieva, Wu 2009). By analogy, the probabilities and parameter update can be evaluated for the case when an agent $i\left(i=1, \cdots N^{B}\right)$ operates in market $B$, and therefore market $B$ is his/her domestic market. Once a trader chooses a market to make a decision, for example, market $B$, he/she then makes the decision using the historical price of market $B$, and the decision table for market $B$. After the trade, he/she updates the score for each strategy in decision table $B$. 
TABLE 2

\begin{tabular}{ccccccc} 
A decision table used for markets A and B \\
\hline \hline Historical & $S_{1}^{A}$ & $S_{2}^{A}$ & $S_{3}^{A}$ & $S_{1}^{B}$ & $S_{2}^{B}$ & $S_{3}^{B}$ \\
\hline$-1,-1$ & 0 & -1 & 0 & 0 & -1 & 1 \\
$-1,0$ & 1 & 1 & 0 & 1 & 1 & 0 \\
$-1,+1$ & 0 & -1 & 1 & 1 & -1 & 1 \\
$0,-1$ & 0 & 0 & 0 & 0 & 0 & 0 \\
0,0 & -1 & 0 & -1 & 1 & 0 & -1 \\
$0,+1$ & 1 & 1 & -1 & 1 & -1 & -1 \\
$+1,-1$ & 0 & 1 & -1 & 0 & 1 & -1 \\
$+1,0$ & -1 & 1 & -1 & 1 & 1 & -1 \\
$+1,+1$ & 0 & -1 & 0 & 0 & -1 & 0 \\
\hline \hline
\end{tabular}

C) Herd traders in a multinational market model: In the multinational market model, herd traders summarize the latest price changes in all markets, and have a tendency to follow the overall market trend (price change) $\zeta$. For example, in the case of two markets, market $A$ and market $B$, the probability $P_{1, t}^{\text {Herd }, A}(i)$ at time $t$ of herd traders in market $A$ to make a buy (1) decision is given as follows:

$$
\begin{aligned}
& P_{1, t}^{\text {Herd }, A}(i)=\frac{\exp \left(\zeta_{t-1}^{A}\right)}{\exp \left(\zeta_{t-1}^{A}\right)+\exp \left(-\zeta_{t-1}^{A}\right)} \\
& \zeta_{t-1}^{A}=\sum_{\text {Market }=A}^{B}\left(\frac{\text { Price }_{t-1}^{\text {Market }}-\text { Price }_{t-2}^{\text {Market }}}{\text { Price }} \text { Market }_{t-2}^{A}\right.
\end{aligned}
$$

Herd traders with high sensitivity, $\tau$, have a high probability of following the overall price change $\zeta$. The probability to make a sell decision $(-1)$ is $P_{-1, t}^{\text {Herd }, A}(i)=1-P_{1, t}^{\text {Herd }, A}(i)$ if we only consider buy and sell decisions (Caporale et al. 2009). However, in order to add one more strategy "hold", we have further introduced into the above formula, the probability of a hold strategy calculated as follows:

$$
P_{0, t}^{\text {Herd }, A}(i)=\frac{1}{1+\left(d\left|\zeta_{t-1}^{A}\right|\right)}
$$

where parameter $d$ controls the sensitivity to price change. The probability of a "buy" decision is revised as:

$$
P_{1, t}^{\text {Herd }, A}(i)=\left(1-P_{0, t}^{\text {Herd }, A}(i)\right) \frac{\exp \left(\zeta_{t-1}^{A}\right)}{\exp \left(\zeta_{t-1}^{A}\right)+\exp \left(-\zeta_{t-1}^{A}\right)}
$$

Correspondingly, the probability of a "sell" decision becomes: 
$p_{-1, t}^{\text {Herd }, A}(i)=1-p_{0, t}^{\text {Herd }, A}(i)-p_{1, t}^{\text {Herd }, A}(i)$

D) Noise traders in a multinational market model: Within a single market, noise traders make decisions to buy, sell or do nothing with different probabilities $p^{b}, p^{s}$, and $p^{h}$, respectively. The probabilities are predefined and remain constant during the whole process of simulation. These settings remain generally the same in the multinational market model; however, we now have noise traders associated with each of the markets. For example, $p_{b}^{\text {Noise }, A}, p_{s}^{\text {Noise }, A}, p_{h}^{\text {Noise, A }}$ are the probabilities for noise traders' decisions in market $A$. For market $B$, the corresponding probabilities are $p_{b}^{\text {Noise }, B}, p_{s}^{\text {Noise }, B}, p_{h}^{\text {Noise }, B}$.

E) An integrated Mixed Game and GP based multinational artificial stock market (IMgGpM)

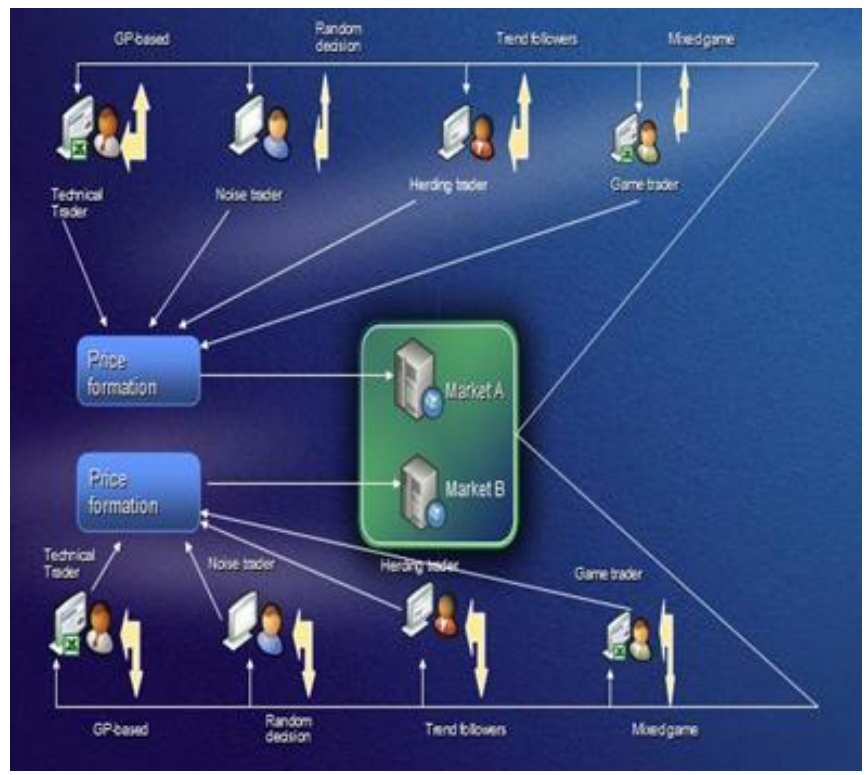

Fig.2. Multinational agent-based model

In the multinational market, as pictured in Figure 2, each type of player makes decisions based on the information from all markets. For example, in market $A$, during each trade, after decisions are made, a new market price is calculated with the price formation formula. The price information of both markets, $A$ and $B$, becomes public information and each player re-evaluates their strategies. If a decision made based on market $B$, a foreign market, is more accurate than that based on market $A$, the domestic market, the player will have a higher probability of choosing information from market $B$ to make a decision in the next trading period. The same rules apply to players in market $B$. The above procedure is a further development of (Caporale, Serguieva, Wu 2008; Caporale, Serguieva, Wu 2009). 


\subsection{GARCH Model and Clayton-Copula for Return Simulation}

\subsubsection{Copula Functions}

Interdependence is a key measure of how two markets are co-related to each other. In our agent-based model, interdependence is used as a criterion for the fitness function which contains a group of parameters to be optimized. For random variables which are normally distributed, linear correlation completely characterizes interdependence. However, the linear correlation coefficient is far less meaningful for nonnormal distributions. Unfortunately, researchers find the data in the area of financial markets does not follow a normal distribution. Given this limitation of the linear correlation coefficient, the use of copula function has become popular in the area of financial mathematics. Given two random variables X, Y, with continuous univariate distributions $\mathrm{F}_{\mathrm{x}} \mathrm{F}_{\mathrm{y}}$, suppose that a continuous joint distribution $\mathrm{H}(\mathrm{x}, \mathrm{y})$ also exists. Then there exists a unique function, called a copula, which maps the pair $\left(\mathrm{F}_{\mathrm{x}}, \mathrm{F}_{\mathrm{y}}\right)$ into $\mathrm{H}(\mathrm{x}, \mathrm{y})$. Crucially, this function can be parameterized independently from the underlying univariate distributions. Different choices of copula functions focus on different aspects of interdependence. The reader is referred to Nelsen (1999) and Cherubini (2004) for more detail on copula functions.

In this paper, we use the Clayton copula to model the interdependence of two markets. Let us define Clayton copula as:

$\Psi(x)=x^{\theta}-1 ; \theta \leq 0 ; \quad H(x, y)=\left(F(x)^{\wedge} \theta+G(y)^{\wedge} \theta-1\right)^{1 / \theta}$

Where $F(x)$ and $G(y)$ are the distribution functions of variable $x$ and $y . \theta$ is the parameter of the Clayton copula family, and $\Psi(x)$ is the generator function. For $\theta=0$ in the Clayton copula, the random variables are statistically independent. The generator function approach can be extended to create multivariate copulas, by simply including more additive terms.

Let $\left(x_{1}, y_{1}\right),\left(x_{2}, y_{2}\right) \ldots\left(x_{n}, y_{n}\right)$ be a set of joint observations from two random variables $X$ and $Y$ respectively. Any pair of observations $\left(x_{i}, y_{i}\right)$ and $\left(x_{j}, y_{j}\right)$ are said to be concordant if $\left(x_{i}-x_{j}\right)\left(y_{i}-y_{j}\right)>0$; otherwise they are said to be discordant, (Nelsen, 2001). The Kendall's coefficient $\mathfrak{G}$ is defined as:

$$
\mathfrak{H}=\frac{\text { (number of concordant pairs) }-(\text { number of discordant pairs) }}{\frac{1}{2} \mathrm{n}(\mathrm{n}-1)}
$$

\subsubsection{GARCH $(1,1)$ Model}

A large number of references (e.g. Engel 1982) reveal that Generalized Auto-Regressive Conditional Heteroskedasticity (GARCH) could successfully model market dynamics and volatility. In this paper, we choose GARCH $(1,1)$ to model both the domestic and foreign market return data series, which are later used as input to calculate the copula. Now, consider a $\operatorname{GARCH}(1,1)$ model:

$r_{t}=\mu+a_{t}$

$a_{t}=\sigma_{t} * \varepsilon_{t}, \varepsilon_{t} \sim N(0,1) \quad$,

$\sigma_{t}^{2}=\alpha_{0}+\alpha_{1} a_{t-1}^{2}+\beta \sigma_{t-1}^{2} \quad$, 
where $r_{t}$ is the return series, $\mu$ is the mean value of $r_{t}, a_{t}$ is the volatility of $r_{t}, \varepsilon_{t}$ is standard normal distribution, and $\alpha_{0}, \alpha_{1}, \beta$ are parameters needed to be estimated. As the returns are conditionally normal, one can use maximum likelihood method to find the parameters.

\subsubsection{Tail Dependence Coefficient}

Tail dependence describes the limiting proportion that one margin exceeds a certain threshold given that the other margin has already exceeded that threshold. Let $X$ and $Y$ be continuous random variables with distribution functions $F$ and $G$, respectively. The upper tail dependence parameter $\lambda_{U}$ is the limit (if it exists) of the conditional probability that $Y$ is greater than the 100t-th percentile of $G$ given that $X$ is greater than the 100t-th percentile of $F$ as $t$ approaches 1, i.e.

$\lambda_{U}=\lim _{t \rightarrow 1^{-}} P\left[Y>G^{-1}(t) \mid X>F^{-1}(t)\right]$

Similarly, the lower tail dependence parameter $\lambda_{L}$ is the limit (if it exists) of the conditional probability that $Y$ is less than or equal to the $100 \mathrm{t}$-th percentile of $G$ given that $X$ is less than or equal to the $100 \mathrm{t}$-th percentile of $F$ as $t$ approaches 0 , i.e.

$\lambda_{L}=\lim _{t \rightarrow 0^{-}} P\left[Y \leq G^{-1}(t) \mid X \leq F^{-1}(t)\right]$

\subsection{Parameter Estimation}

\subsubsection{Parameters for GARCH-Copula Model}

After we transform the return series data into the uniform distribution with the following formula:

$$
\begin{aligned}
& P\left(R_{t+1} \leq r_{t+1}\right)=P\left(R_{t+1}-\mu \leq r_{t+1}-\mu\right)=P\left(a_{t+1} \leq r_{t+1}-\mu\right) \\
& =P\left(\sigma_{t+1} * \varepsilon_{t+1} \leq r_{t+1}-\mu\right)=P\left(\sqrt{\alpha_{0}+\alpha_{1} a_{t}^{2}+\beta \sigma_{t}^{2}} \varepsilon_{t+1} \leq r_{t+1}-\mu\right) \\
& =P\left(\varepsilon_{t+1} \leq \frac{r_{t+1}-\mu}{\sqrt{\alpha_{0}+\alpha_{1} a_{t}^{2}+\beta \sigma_{t}^{2}}}\right)=\mathrm{N}\left(\frac{r_{t+1}-\mu}{\sqrt{\alpha_{0}+\alpha_{1} a_{t}^{2}+\beta \sigma_{t}^{2}}}\right)
\end{aligned}
$$

where $R_{t+1}$ is the probability distribution of the asset's return in time $\mathrm{t}+1$, and we then estimate the Clayton copula's parameters.

According to (Nelsen1999), the relationship between Kendal's tau $(\mathfrak{H})$ and $\operatorname{theta}(\theta)$ is :

$\theta=\frac{2 \mathfrak{H}}{1-\mathfrak{H}}$

and $\theta$ is the parameter we need to calculate. The tail dependence coefficient of Clayton copula is $2^{-\frac{1}{\theta}}$ (Nelsen1999).

\subsubsection{Parameter Estimation}

The objective of our model is to simulate the contagion effect present during the South Asian crisis of 1997. To achieve this purpose, we consider the following requirements established in Gou (2006). 
A) The memory size of both types of game players must be larger than two, and the memory size of majority game players should be less than that of minority players, while the time window for majority game players cannot be larger than that for minority players.

B) The plots of return distributions should look similar.

C) The median of the local volatilities of simulation results should be similar to the target real market.

D) Co-movements with the market from which the crisis originated should look similar.

Following these constraints, first the general parameters for market participants are determined. They are technical, game, herd, and noise traders, and their number is denoted as $N_{T}, N_{F}, N_{H}, N_{N}$, respectively. Besides these we need values for the remaining parameters to be able to simulate heterogeneous markets. The additional parameters include $\sigma$ in formula (1), sensitivity factor $\tau$ in formula (11), memory size $m$, number of strategies $k$ included in a decision table for game players, and time period $T$ for technical players. Empirical studies of price fluctuation for various financial assets have shown that stock return deviate from the Gaussian distribution and are characterized with fat tails. Inappropriate proportions of $N_{T}, N_{F}, N_{H}, N_{N}$ may lead to results which make no sense. For example, owing to the different indices and trading volumes in each market, we should model a proper scale factor $\lambda$ to ensure that the median of local volatilities is similar to that of the real target market. Furthermore, according to the definition given by Forbes and Rigobon (2002), financial contagion occurs when the correlation between the original market and the affected market increases significantly between a period of relative stability and a period of crisis. Taking the Asian financial crisis of 1997 as an example, the first period corresponds to the pre-crisis phrase, and the second period corresponds to the crisis phase. The correlation during the pre-crisis phase between Thailand and South Korea is -0.64 , while during the crisis phase it becomes 0.92 , (see Table 3 ). This confirms the expectation of a significant increase in the co-movement of markets. Therefore, we have to identify a proper sensitivity parameter $\tau$ in formula (11).

In order to measure the performances of parameter configurations, we compare the Clayton copula generated by the artificial financial market with the real Clayton copula of the target market. We repeat the simulation for the same parameter configuration several times and the mean value of those repeated simulations is regarded as the final fitness value of the corresponding parameter configuration. The number of times a single parameter configuration is used in simulation is denoted by $\varrho$, the parameter of the Clayton copula of the real target market $B$ and the original market $A$ at time $t$ is denoted by $\tilde{\theta}_{t}^{R E L, A B}$, and the Clayton copula parameter of the $i$ th time repetitive simulation is denoted by $\theta_{t}^{\operatorname{SIM}, A B}(i), i=1, \ldots \ldots \varrho$. Thus the fitness function for the individual parameter configuration to be minimized is given by

$f\left(p^{S I M, B}\right)=\sum_{i=1}^{\varrho} \sum_{t}\left(\frac{\left|\widetilde{\theta}_{t}^{R E L, A B}-\theta_{t}^{S I M, A B}(i)\right|}{\varrho^{\cdot t}}\right)$

\section{Simulations and Analysis}

We apply an evolutionary optimization technique for our model and simulate the Asian fcrisis of 1997. 


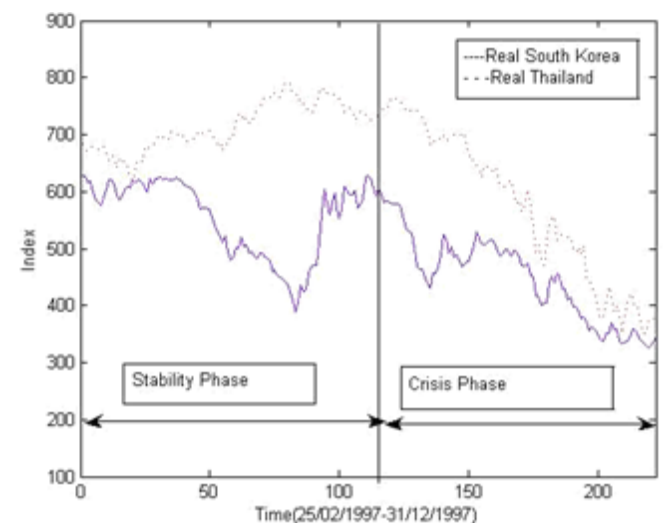

Fig 3: Indices of Thailand's and South Korea's stock market, from $25 / 02 / 1997$ to $31 / 12 / 1997$.

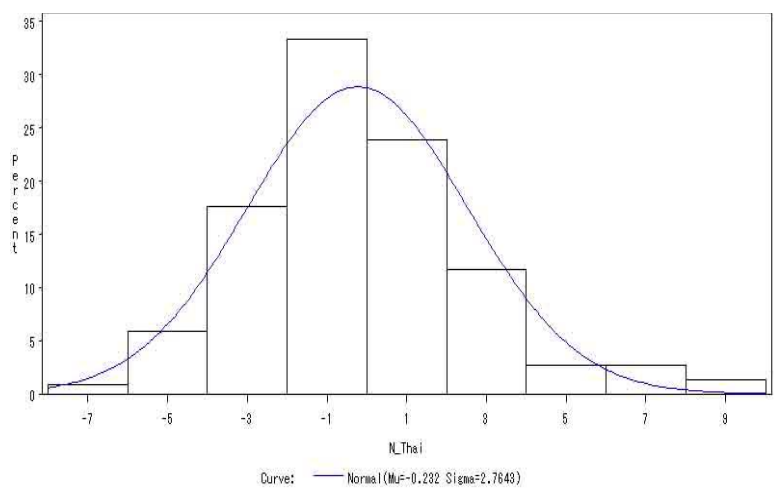

Fig.4: Distribution of Thailand's real market index, from $25 / 02 / 1997$ to $31 / 12 / 1997$

The crisis originated in Thailand, and affected almost all the financial markets in Asia, including Hong Kong, Malaysia, and South Korea. We simulate the index of South Korea to target the real market over 222 trading days, from $25 / 02 / 1997$ to $31 / 12 / 1997$ in relation to the movement of the Thailand stock market. This time period is divided into two parts: the first 111 days constitute the pre-crisis phase, and the last 111 days correspond to the crisis phase. The real market and simulated values for South Korea are set out in Table 3, and the parameters for simulating this target market are shown in Table 4.

TABLE 3: Result for South Korea

\begin{tabular}{lll}
\hline \hline Target Value & Real & Simulated \\
\hline Kurtosis of daily return distribution & 3.08 & 8.38 \\
Volatility & 63.7 & 54.1 \\
Correlation coefficient in pre-crisis & -0.64 & -0.74 \\
Correlation coefficient in crisis & 0.92 & 0.97 \\
Tail dependence coefficient before & 0.4557 & 0.1111 \\
Tail dependence coefficient during & 0.6655 & 0.8055 \\
\hline \hline
\end{tabular}

TABLE 4: Symbols and parameters for South Korea

\begin{tabular}{ccc}
\hline \hline Symbol & Represents & Parameter \\
\hline$N$ & Total number of players & 180 \\
$N_{T}$ & Technical traders & 30 \\
$N_{F}$ & Game traders & 60 \\
$N_{H}$ & Herding traders & 80 \\
$N_{N}$ & Noise traders & 10 \\
$K 1$ & Majority game strategies & 30 \\
$K 2$ & Minority game strategies & 60 \\
$T$ & Time period for technical traders & 5 \\
$M 1$ & Memory size of minority game players & 10 \\
$M 2$ & Memory size of majority game players & 20 \\
$\tau$ & sensitivity factor for herding traders & 30 \\
$\sigma$ & Scale factor used calculate price & 4 \\
\hline \hline
\end{tabular}


Next, Figures 5 and 6 present the South Korea simulation of daily returns and the median of local volatility from 25/02/1997 to 31/12/1997, or 222 trading days.

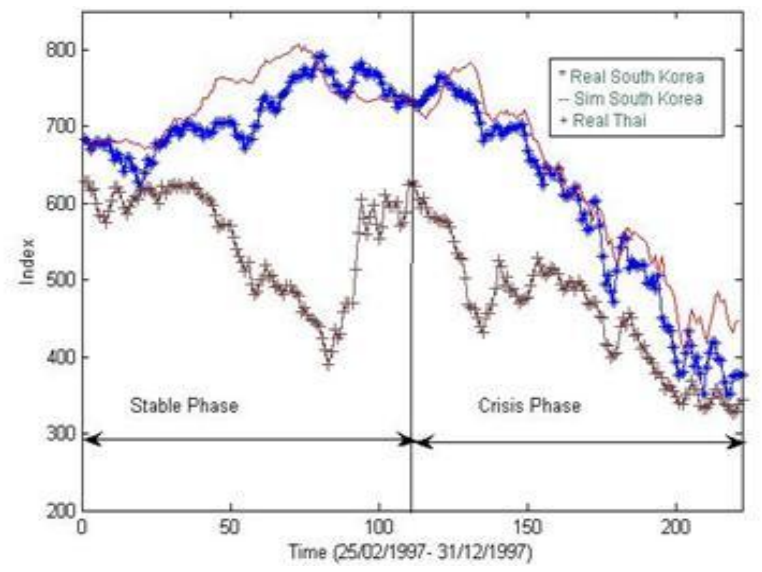

Fig. 5: A comparison of the simulated and real market indices of South Korea and the real index of Thailand

The correlation coefficient of the simulated South Korea series and Thailand's actual series is -0.74 in the pre-crisis phase and rises to 0.97 during the crisis phase. After the crisis happened, the lower tail dependence increased from 0.1111 to 0.8055 , which means big losses in extreme cases become closely related. During the pre-crisis phase, Thailand suffers an initial tumble and then returns to normal levels. South Korea does not follow it, showing that it has not been affected at this point. In our model, both the real and simulated correlation coefficients of the two markets in the pre-crisis phase are relatively stable. Furthermore our model successfully picks up the increase in the correlation coefficient during the crisis period. The simulated index of both the stable phase and crisis phase match the real data very well.
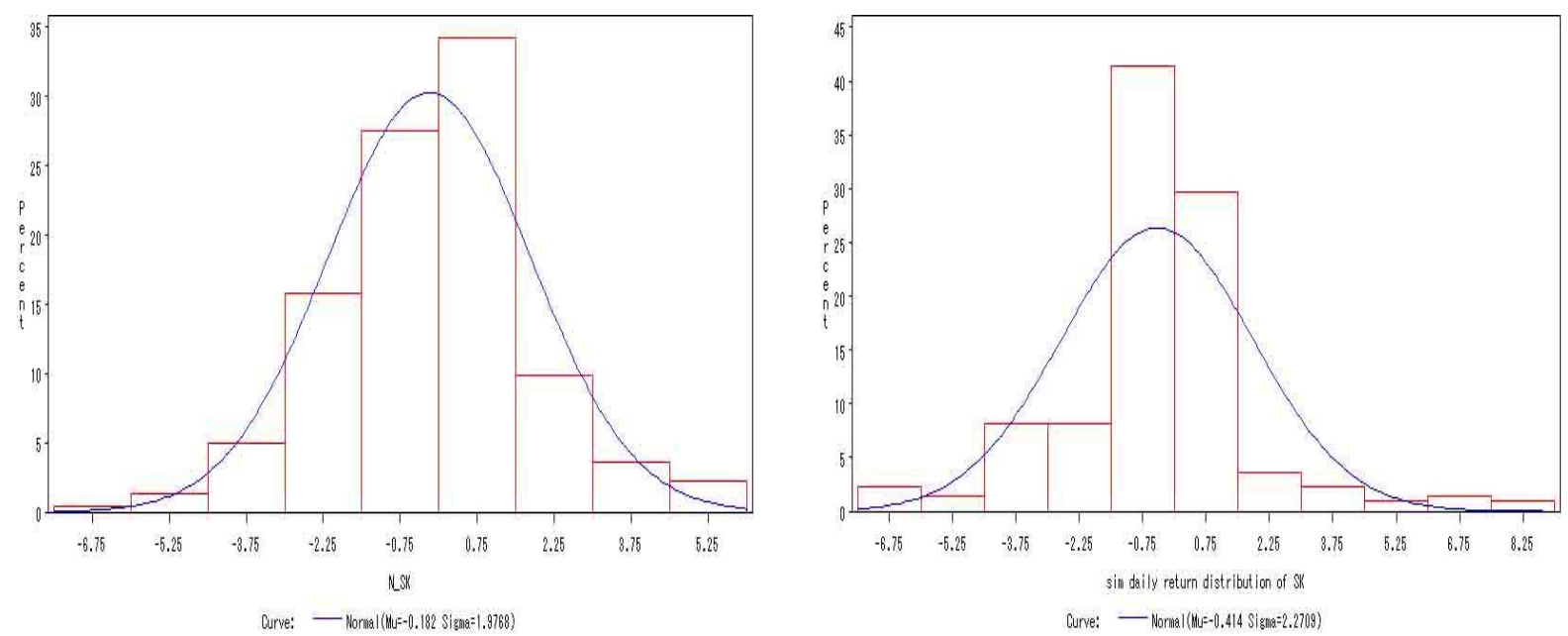

Fig.6: South Korea's market index - real (left graph) and simulated (right graph). 
The predicting ability of this model is also fairly strong. As we move the time window forward for 37 trading days, the real tail dependence, increased from 0.4557 to 0.4728 , which means the contagion was happening. The tail dependence $2^{-\frac{1}{\theta}}$ is calculated through the coefficient of the Clayton copula $\theta$ from fotmulas (21) and (16). Our model further shows an increase from 0.1111 to 0.2055 in the simulated data, which means we can predict the contagion to some extent.

\section{Conclusions}

In this paper we develop an agent-based financial market model comprising four types of traders - technical, game, herd and noise traders - who make buy, hold and sell decisions, based on different characterising strategies. The developed model also represents multiple markets rather than a single market, in order to simulate how financial contagion happens and observe the evolution of interactions between markets. Using real data for Thailand, where the Asian crisis of 1997 originated, and simulating the movements of the affected market of South Korea, we observe a significant increase in the correlation between markets during the crisis phase, as compared to the pre-crisis phase. Thus the model is capable of successfully simulating that important characteristic of contagion. Several features of our model contribute to that capability. First, the model involves agents with different type of behaviour and different strategies even with the same type of behaviour, which provides for a richer, complex, and more realistic simulated system. Second, the strategies incorporate information from multiple rather than a single market, thus the simulated market links emerged through the underlying behaviours of agents. Third, instead of following previous studies and using a linear measure of how markets are co-related with each other, we introduce the nonlinear Clayton copula function to measure non-linear interdependence between non-normally distributed financial data. That measure captures more realistic cross-market links, and it is used in the fitness function when optimizing our model parameters. Finally, we have made initial steps towards investigating the predictive ability of the model through moving the time window and measuring and comparing tail dependence.

Future research will further allow agents to change between the four types of trading behaviour temporarily, thus better reflecting the reality that during a crisis period more people tend to follow trends rather than remain rational. An underlying psychology analysis can also be incorporated into the modelled behavioural types. Additionally, the mixed game can be extended into an evolutionary game to shed further light on the game-traders behaviour. Finally, the Clayton copular could be replaced by the Gumbel-Clayton Copula which is good in capturing both upper tail and lower tail dependences.

\section{References}

1. B.H. BOYER., 1997 "Pitfalls in tests for changes in correlations", International Finance Discussion Paper, Board of Governors of the Federal Reserve System.

2. G.M Caporale, A Cipollini, N. Spagnolo, N, 2005 "Testing for contagion: a conditional correlation analysis", Journal of Empirical Finance, vol. 12, no. 3, pp. 476-489. 
3. G.M Caporale, A Serguieva, H Wu., 2009 "Financial contagion: evolutionary optimization of a multinational agent-based model", Intelligent System in Accounting, Finance and Management, vol. 16, no 12, 2009, pp. 111-126.

4. U. Cherubini., E Luciano,., W Vecchiato,.,(2004) “Copula Methods in Finance”, Publisher: John Wiley $\&$ Sons, Ltd.

5. A, G Cipollini. G Kapetanios, 2009, Forecasting financial crises and contagion in Asia using dynamic, factor analysis, Journal of Empirical Finance 16, 188-200.

6. R Cont, J Bouchaud, 2000, "Herd behaviour and aggregate fluctuations in financial markets," Macroeconomic Dynamics, vol. 4, no. 2, pp. 170-196.

7. De Long., J Bradford, A Shleifer, L Summers, And R.J. Waldmann, 1990 "Noise trader risk in financial markets,” Journal of Political Economy, vol. 98, no. 4, pp: 703-738.

8. G.V Durrleman., A Nikegehbali., T Roncalli., (2000) "Which copula is the right one", Groupe de Recherche Opérationnelle, Crédit Lyonnais, France working paper, pp: 44-46.

9. R.F Engle., (1982) "autoregressive conditional heteroskdesticity with estimates of the variance of United Kingdom inflation”, Econometrica, pp: 987-1007.

10. J.D FARMER, 2006 "Market force, ecology, and evolution," Industrial and Corporate Change, vol. 11, pp. 895-953.

11. K. Forbes, R RigOBON 2002, "No contagion, only interdependence: measuring stock market co-movements," Journal of Finance, vol. 57, no. 5, pp. 2223-2261.

12. I, J Giardina. J.P Bouchaud 2003, "Bubbles, crashes and intermittency in agent based market models," The European Physical Journal B -Condensed Matter, vol. 31, pp. 421-437.

13. C. Gou, 2006, "The simulation of financial markets by an agent-based mixgame model", Journal of Artificial Societies and Social Simulation, vol.9, no. 3, pp. 321-328.

14. P Jefferies, M. Hart, P. Hui, N. Johnson, 2001, "From market games to real-world markets," The European Physical Journal B - Condensed Matter and Complex Systems, vol. 20, pp. 493-501.

15. G KAMINSKY, S LizONDO, AND C ReINHART, 1998, "Leading indicators of currency crisis," IMF Staff Papers, International Monetary Fund, vol. 45, no. 1.

16. G KAMINSKY, 1999, "Currency and banking crises - the early warning of distress," IMF Working Papers, International Monetary Fund, no. 99/178.

17. M. King, S. Wadhwani, 1990, "Transmission of volatility between stock markets," Review of Financial Studies, vol. 3, no. 1, , pp. 5-33.

18. B. LebARON, 2006, "Agent-based computational finance: suggested readings and early research," Journal of Economic Dynamics and Control, vol. 23, no. 9-10, pp. 1487-1516.

19. S. MARTINEZ-JARAMILlo, E. TSAnG, 2009, "A heterogeneous and co-evolutionary GP-based financial market," IEEE Transaction on Evolutionary Computation, vol. 63, no. 1, pp. 1105-1142.

20. R.B. NELSEN., (1999) “An Introduction to Copulas”, Springer Science Business Media, Inc., ISBN-10: 0-38728659-4, ISBN-13: 978-0387-28659-4.

21. R.B. Nelsen,. (2001), "Kendall tau metric", in Hazewinkel, Michiel, Encyclopaedia of Mathematics, Springer, ISBN 978-1556080104,

22. J.C RodrigueZ, 2007, Measuring Financial Contagion: A Copula Approach, Journal Of Empirical Finance 14, 401-423. 
23. M TANaKa-Yamawaki, S ToKuOKa, 2006, "Minority Game As A Model For The Artificial Financial Markets," Proceedings of 2006 IEEE Congress on Evolutionary Computation, pp. 2157-2162.

24. Van Valen Leigh, 1973, “A New Evolutionary Law,” Evolutionary Theory, Vol. 1, pp. 1-30.

25. J YAng, D.A Bessler, 2006, Contagion Around The October 1987 Stock Market Crash, European Journal Of Operational Research 184 (2008) 291-310.

26. A. Serguieva, H. Wu. 2008, Computational Intelligence In Financial Contagion Analysis , Intel Journal Complex System,No,2229,Pp:1-12,New England Complex System Institute .

27. G.M Caporale, A Serguieva, H Wu., 2008, A Mixed Game Agent Based Model For Simulating Financial Contagion, Proceeding Of 2008 IEEE World Congress On Computational Intelligence, Hong Kong, Pp:3421.

28. F.Liu, A.Serguieva,P.DAte,2010, A Mixed-Game and Co-Evolutionary Genetic Programming Agent-Based Model of Financial Contagion, Proceeding Of 2010 IEEE World Congress Of Evolutionary Computation, Barcelona, 